\title{
ABSTRACT REFLECTIONS AND COXETER GROUPS
}

\author{
DAVID M. GOLDSCHMIDT ${ }^{1}$
}

\begin{abstract}
A notion of "abstract reflection" is introduced and used to characterize the Coxeter groups. Several known results on Coxeter groups are obtained as easy corollaries.
\end{abstract}

1. Introduction. Let $I$ be a set of indices, and let $\mathbf{Z}^{+}$denote the positive integers. Recall that a Coxeter matrix $M=\left(m_{i j}\right)_{i, j \in I}$ is a doubly indexed subset of $\mathbf{Z}^{+} \cup\{\infty\}$ such that $m_{i i}=1$ for all $i \in I$. A Coxeter system (of type $M)$ is an indexed subset $S=\left\{s_{i}\right\}_{i \in I}$ of some group such that:

(1) $\left(s_{i} s_{j}\right)^{m_{i j}}=1$ for all $i, j \in I$;

(2) the relations (1) are a presentation for the subgroup $\langle S\rangle$ generated by $S$.

To eliminate obvious redundancies, we will always assume $m_{i j}=m_{j i}>2$, even though this is not really necessary. Coxeter systems were studied systematically by Coxeter [2] and later by Tits [3] and Bourbaki [1]. They seem to play a fundamental role in group theory.

In this semi-expository note, we introduce a notion of "abstract reflection" and use it to characterize Coxeter systems. Since the argument is direct and does not depend on any previous characterizations, we show how several known results can be obtained as easy corollaries. We also observe that the result can be restated in the language of graph theory.

2. Abstract reflections. Let $w$ be a permutation on a set $\Omega$. For a subset $A \subseteq \Omega$ we let $A^{w}$ denote its image under $w$. An abstract reflection on $\Omega$ is a pair $(s, D(s))$ where $s$ is a permutation of $\Omega$ of order $2, D(s) \subseteq \Omega$, and $D(s) \cap D(s)^{s}=\varnothing$. We define $E(s)=D(s)^{s}$.

Given a set of abstract reflections $\{(s, D(s)) \mid s \in S\}$ we define

$$
D(S)=\bigcap_{s \in S} D(s)
$$

and we say that $S$ is a system of reflections if $D(S) \neq \varnothing$. We say that a system of reflections $S$ is proper if, given any $s \in S$ and $w \in\langle S\rangle$, one of the following conditions holds:

(i) $D(S)^{w} \subseteq D(s)$,

(ii) $D(S)^{w} \subseteq E(s)$ and $l_{S}(w s)<l_{S}(w)$.

Received by the editors April 21, 1977.

AMS (MOS) subject classifications (1970). Primary 20F05, 20H10, 20H15, 50B30, 50B35, 50C05, 22E40, 30A58, 17B20, 05C20, $05 \mathrm{C} 25$.

'Partially supported by NSF Grant MCS 76-00163 and the Alfred P. Sloan foundation. 
Here, $l_{S}(w)$ denotes the length of a shortest word in the generators $S$ which is equal to $w$ in $\langle S\rangle$. Finally, we shall say that a system of reflections is pairwise proper if every pair of distinct elements of $S$ is itself a proper set of reflections.

3. A characterization of the Coxeter groups. In this section, we assume throughout that $S$ is a system of pairwise proper reflections on some set $\Omega$, with $D=D(S)$. Suppose that $s, t$ are distinct elements of $S$ and $w \in\langle S\rangle$. We say that an expression of the form $w=w_{0} w_{1}$ is a $\langle s, t\rangle$-decomposition of $w$ if the following conditions are satisfied:

(1) $w_{0} \in\langle S\rangle$ and $1 \neq w_{1} \in\langle s, t\rangle$,

(2) $l_{s}(w)=l_{s}\left(w_{0}\right)+l_{\langle s, t\rangle}\left(w_{1}\right)$.

(3.1) $S$ is a proper system of reflections.

Proof. Let $s \in S$, and $w \in\langle S\rangle$. We want to show that either $D^{w} \subseteq D(s)$ or that $D^{w} \subseteq E(s)$ and $l_{s}(w s)<l_{s}(w)$. Since this is trivial for $w=1$, we proceed by induction on $l_{S}(w)$. Write $w=\tilde{w}_{0} t$ for some $t \in S$ where $l_{S}\left(\tilde{w}_{0}\right)<$ $l_{s}(w)$. If $t=s$, induction yields $D^{\tilde{w}_{0}} \subseteq D(s)$ since $l_{s}\left(\tilde{w}_{0} s\right)=l_{s}(w)>l_{s}\left(\tilde{w}_{0}\right)$. Then $D^{w} \subseteq E(s)$ and there is nothing more to prove. So we may assume that $t \neq s$. Hence, $w=\tilde{w}_{0} t$ is a $\langle s, t\rangle$-decomposition of $w$. Let $w=w_{0} w_{1}$ be a $\langle s, t\rangle$-decomposition of $w$ with $l_{s}\left(w_{0}\right)$ as small as possible. Then $\left.l_{s}\left(w_{0} s\right)\right\rangle$ $l_{s}\left(w_{0}\right)$, since otherwise we could write $w=\left(w_{0} s\right)\left(s w_{1}\right)$, obtaining a $\langle s, t\rangle$ decomposition which violates the minimality of $l_{s}\left(w_{0}\right)$. Therefore, we have $D^{w_{0}} \subseteq D(s)$ by induction, and similarly, $D^{w_{0}} \subseteq D(t)$. Hence,

$$
D^{w}=D^{w_{0} w_{1}} \subseteq[D(s) \cap D(t)]^{w_{1}}=D(s, t)^{w_{1}} .
$$

However, since $\{s, t\}$ is proper and $w_{1} \in\langle s, t\rangle$, we have either

(i) $D(s, t)^{w_{1}} \subseteq D(s)$, or

(ii) $D(s, t)^{w_{1}} \subseteq E(s)$ and $l_{\langle s, t\rangle}\left(w_{1} s\right)<l_{\langle s, t\rangle}\left(w_{1}\right)$, and combining these with (3.2) easily completes the proof.

(3.3) Suppose $s, t \in S, w \in\langle s, t\rangle$, and $w=s_{1} s_{2} \cdots s_{n}$, where $s_{i} \in S(1 \leqslant i$ $\langle n)$ and $n=l_{s}(w)$. Then $s_{i} \in\{s, t\}$ for all $i$.

Proof. Let $u \in S-\{s, t\}$. We argue by induction on $l_{\langle s, t\rangle}(w)$ that $D^{w} \subseteq$ $D(u)$. Since the case $w=1$ is trivial, we may choose notation so that $w=w_{0} t$ with $l_{\langle s, t\rangle}\left(w_{0}\right)<l_{\langle s, t\rangle}(w)$. Then

$$
D^{w_{0}} \subseteq[D(s) \cap D(t)]^{w_{0}} \subseteq D(s, t)^{w_{0}} \subseteq D(t),
$$

the last inclusion being a consequence of our hypothesis that $\{s, t\}$ is proper. By induction we have $D^{w_{0}} \subseteq D(u)$ and, hence,

$$
D^{w}=D^{w_{0} t} \subseteq[D(u) \cap D(t)]^{t}=D(u, t)^{t} \subseteq D(u),
$$

where again the last inclusion follows because $\{u, t\}$ is proper and $t \neq u$.

However, since $l_{s}\left(w s_{n}\right)<l_{s}(w)$, we obtain $D^{w s_{n}} \subseteq D\left(s_{n}\right)$ from (3.1) and, therefore, $D^{w} \subseteq E\left(s_{n}\right)$. By the above it follows that $s_{n} \in\{s, t\}$, and then we 
get $s_{i} \in\{s, t\}$ for $1<i<n$ by an obvious induction argument.

Now for each pair $s, t \in S$, let $m_{s t}$ be the order of $s t$ in $\langle S\rangle\left(m_{s t}=\infty\right.$ is allowed).

(3.4) Suppose that $f$ is a function from $S$ to a group $G$ such that $(f(s) f(t))^{m_{\text {t }}}$ $=1$ for all $s, t \in S$. Then $f$ extends (uniquely) to a homomorphism $\hat{f}:\langle S\rangle \rightarrow G$. Hence, $S$ is a Coxeter system.

Proof. Let $w \in\langle S\rangle$ with $l_{S}(w)=n$ and suppose that there exist (not necessarily distinct) elements $s_{1}, s_{2}, \ldots, s_{n}, s_{1}^{\prime}, s_{2}^{\prime}, \ldots, s_{n}^{\prime} \in S$ such that

$$
s_{1} s_{2} \cdots s_{n}=w=s_{1}^{\prime} s_{2}^{\prime} \cdots s_{n}^{\prime} \text {. }
$$

Then we argue by induction on $n$ that

$$
f\left(s_{1}\right) f\left(s_{2}\right) \cdots f\left(s_{n}\right)=f\left(s_{1}^{\prime}\right) f\left(s_{2}^{\prime}\right) \cdots f\left(s_{n}^{\prime}\right) .
$$

The case $n=1$ is trivial, and the induction hypothesis implies that $f$ extends uniquely to a function

$$
\hat{f}:\left\{w \in\langle S\rangle \mid l_{s}(w)<n\right\} \rightarrow G
$$

such that if $l_{s}\left(w_{1}\right)+l_{s}\left(w_{2}\right)<n$, then

$$
\hat{f}\left(w_{1} w_{2}\right)=\hat{f}\left(w_{1}\right) \hat{f}\left(w_{2}\right) .
$$

In particular, (3.5) implies (3.4).

Suppose first that $w \in\left\langle s_{n}, s_{n}^{\prime}\right\rangle$. Then $\left\{s_{i}, s_{i}^{\prime}\right\} \subseteq\left\{s_{n}, s_{n}^{\prime}\right\}$ for all $i$ by (3.3). But it is an easy exercise to verify that the relation

$$
\left(s_{n} s_{n}^{\prime}\right)^{m_{s_{n} s_{n}}=1}
$$

is a presentation for $\left\langle s_{n}, s_{n}^{\prime}\right\rangle$. Hence, (3.5) is an immediate consequence of the hypothesis

$$
\left(f\left(s_{n}\right) f\left(s_{n}\right)\right)^{m_{n_{n} s_{k}}}=1 .
$$

Returning to the general case, then, we may henceforth assume that $w \notin\left\langle s_{n}, s_{n}^{\prime}\right\rangle$. We may also assume that $s_{n} \neq s_{n}^{\prime}$, since otherwise induction applies trivially. Put $\hat{w}_{0}=s_{1} s_{2} \cdots s_{n-1}, \hat{w}_{0}^{\prime}=s_{1}^{\prime} s_{2}^{\prime} \cdots s_{n-1}^{\prime}$. Then $\hat{f}$ is defined at $\hat{w}_{0}$ and $\hat{w}_{0}^{\prime}$, and (3.5) can be rewritten

$$
\hat{f}\left(\hat{w}_{0}\right) \hat{f}\left(s_{n}\right)=\hat{f}\left(\hat{w}_{0}^{\prime}\right) \hat{f}\left(s_{n}^{\prime}\right) .
$$

Assume, by way of contradiction, that this is false, and choose a pair of $\left\langle s_{n}, s_{n}^{\prime}\right\rangle$ decompositions $w_{0} w_{1}=w=w_{0}^{\prime} w_{1}^{\prime}$ of $w$ such that

(a) $w_{0} \neq 1 \neq w_{0}^{\prime} \quad$ (so that $\hat{f}$ is defined at $w_{0}, w_{0}^{\prime}, w_{1}, w_{1}^{\prime}$ ),

(b) $\hat{f}\left(w_{0}\right) \hat{f}\left(w_{1}\right) \neq \hat{f}\left(w_{0}^{\prime}\right) \hat{f}\left(w_{1}^{\prime}\right)$,

(c) $l_{S}\left(w_{0}\right)+l_{S}\left(w_{0}^{\prime}\right)$ is minimal subject to (a) and (b).

We claim that $D^{w_{0}} \subseteq D\left(s_{n}\right) \cap D\left(s_{n}^{\prime}\right)$, for if not, (3.1) yields $l_{s}\left(w_{0} t\right)<$ $l_{s}\left(w_{0}\right)$ for $t=s_{n}$ or $t=s_{n}^{\prime}$. Since we are assuming $w \notin\left\langle s_{n}, s_{n}^{\prime}\right\rangle$, we have $w_{0} t \neq 1$ and $l_{s}\left(t w_{1}\right)<n$, whence

$$
\hat{f}\left(w_{0} t\right)=\hat{f}\left(w_{0}\right) f(t), \hat{f}\left(t w_{1}\right)=f(t) \hat{f}\left(w_{1}\right),
$$


and

$$
\hat{f}\left(w_{0} t\right) \hat{f}\left(t w_{1}\right)=\hat{f}\left(w_{0}\right) \hat{f}\left(w_{1}\right) \neq \hat{f}\left(w_{0}^{\prime}\right) \hat{f}\left(w_{1}^{\prime}\right) .
$$

But then $\left(w_{0} t\right)\left(t w_{1}\right)=w=w_{0}^{\prime} w_{1}^{\prime}$ is a pair of $\left\{s_{n}, s_{n}^{\prime}\right\}$-decompositions which satisfy (3.6)(a) and (b) and therefore violate the minimality of $l_{S}\left(w_{0}\right)+l_{s}\left(w_{0}^{\prime}\right)$. We conclude that $D^{w_{0}} \subseteq D\left(s_{n}\right) \cap D\left(s_{n}^{\prime}\right)$.

By the same argument, we obtain $D^{w_{0}^{\prime}} \subseteq D\left(s_{n}\right) \cap D\left(s_{n}^{\prime}\right)$ and therefore,

$$
\left[D\left(s_{n}\right) \cap D\left(s_{n}^{\prime}\right)\right]^{w_{1}} \cap\left[D\left(s_{n}\right) \cap D\left(s_{n}^{\prime}\right)\right]^{w_{1}^{\prime}} \supseteq D^{w} \neq \varnothing .
$$

It follows that $D\left(s_{n}, s_{n}^{\prime}\right)^{w_{1}^{\prime} w_{1}^{-1}} \cap D\left(s_{n}, s_{n}^{\prime}\right) \neq \varnothing$, which, since $\left\{s_{n}, s_{n}^{\prime}\right\}$ is proper, implies that $w_{1}^{\prime} w_{1}^{-1}=1$. This is an obvious contradiction to (3.6)(b), which completes the proof of (3.5) and hence, of (3.4).

To complete our characterization of Coxeter systems, it is only necessary to show that every Coxeter system can be realized as a set of pairwise proper abstract reflections on some set $\Omega$. But, in fact, it is well known that every Coxeter system can be realized as a set of pairwise proper bona fide reflections on a real vector space, relative to a canonically determined symmetric (but not necessarily positive definite) bilinear form. Since this follows easily from what we have already done, we will include an explanation for the sake of completeness.

Let $M=\left(m_{i j}\right)_{i, j \in I}$ be a Coxeter matrix and let $\left\{e_{i}\right\}_{i \in I}$ be a basis for a real vector space $V$. Let $($,$) be the unique bilinear form on V$ for which $\left(e_{i}, e_{j}\right)=-\cos \left(\pi / m_{i j}\right)$, where $\pi / \infty=0$, and for each $i \in I$, define a linear transformation $s_{i}: V \rightarrow V$ via the formula

$$
s_{i}(v)=v-2 \cdot\left(v, e_{i}\right) e_{i} \text { for all } v \in V .
$$

Let $\Omega=V^{*}$ be the space of all linear functionals on $V$, let $s_{i}^{*}: V^{*} \rightarrow V^{*}$ be the adjoint map, and let $D\left(s_{i}^{*}\right)=\left\{f \in V^{*} \mid f\left(e_{i}\right)>0\right\}$. It is then a routine exercise in linear algebra to show that $S=\left\{s_{i}^{*}\right\}_{i \in I}$ is a set of abstract reflections on $\Omega$, that $D(S) \neq \varnothing$, and that $S$ is pairwise proper (this last easily reduces to the case $|I|=2$ where it is an elementary fact about the plane).

Finally, we remark that (3.1) shows that every orbit of $\langle S\rangle$ on $\Omega$ which meets $D(S)$ is permutation isomorphic to the regular representation by an isomorphism which maps $D(s)$ to $\left\{w \in\langle S\rangle \mid l_{s}(w s)>l_{s}(w)\right\}$. Hence, there is essentially only one way in which a Coxeter system may be realized as a set of abstract reflections.

4. A restatement and a known result. We first observe that the results of the previous section can be restated in the language of graph theory. Namely, let $\mathcal{G}=(\Omega, E)$, where $\Omega$ is a vertex set and $E \subseteq \Omega \times \Omega$ is an antisymmetric set of directed edges. For $\alpha \in \Omega$, let $E_{\alpha}=(\{\alpha\} \times \Omega \cup \Omega \times\{\alpha\}) \cap E$ be the set of edges adjacent to $\alpha$. We say that $\alpha$ is a source if $\Omega \times\{\alpha\} \cap E=\varnothing$. Suppose that $f: E \rightarrow S$ is a map to some set $S$ which restricts to a bijection on each $E_{\alpha}$. Then we say that $\mathcal{G}$ is a directed graph, edge-labeled by $S$ (via $f$ ). For $S_{0} \subseteq S$, we put $E_{S_{0}}=f^{-1}\left(S_{0}\right)$ and $\mathcal{G}_{S_{0}}=\left(\Omega, E_{S_{0}}\right)$. Then $\mathcal{G}_{S_{0}}$ is edge-labeled by $S_{0}$. 
Given a Coxeter system $S$ of type $M$, put $\Omega=\langle S\rangle$ and define, for each $s \in S$,

$$
E_{s}=\left\{(w, w s) \mid w \in \Omega, l_{s}(w s)>l_{s}(w)\right\} .
$$

Let $E=\cup_{s \in S} E_{s}$ and let $\mathcal{G}(M)=(\Omega, E)$, then $\mathcal{G}(M)$ is a directed graph, edge-labeled by $S . \mathcal{G}(M)$ is just the adjacency graph of the "Coxeter complex," directed by distance (see [3, p. 4]). When $S$ has cardinality 2 , we denote by $P_{n}$ the directed polygon $\mathcal{G}\left(\begin{array}{ll}1 & n \\ n & 1\end{array}\right)$.

(4.1) Suppose that $\mathcal{G}$ is a directed graph, edge-labeled by $S$, with at least one source. Furthermore, suppose that for each pair $s, t \in S$ there exists $m_{s t} \in \mathbf{Z}^{+}$ $\cup\{\infty\}$ such that every connected component of $\mathcal{G}_{\{s, t\}}$ is isomorphic to $P_{m_{3 i}}$. Put $M=\left(m_{s t}\right)_{s, t \in S}$; then $\mathcal{G}$ is isomorphic to $\mathcal{G}(M)$.

Proof. For each $s \in S$, let $\hat{s}$ be the unique permutation of $\Omega$ whose orbits are the connected components of $\mathcal{G}_{\{s\}}$, and let $D(\hat{s})=\left\{\alpha \in \Omega \mid\left(\alpha, \alpha^{\hat{s}}\right) \in E_{s}\right\}$. Then $\hat{S}=\{\hat{s} \mid s \in S\}$ is a system of reflections because $\mathcal{G}$ has a source, and is pairwise proper by hypothesis. The result follows.

A useful characterization of Coxeter groups in the study of $B N$-pairs [3] is the following result from Bourbaki [1]:

(4.2) Let $S$ be a set of involutions in a group $G$. Suppose that for each $s \in S$, there exists a subset $D(s) \subseteq\langle S\rangle$ such that:

(1) $1 \in D(s)$ for all $s \in S$;

(2) $D(s), \cap D(s) s=\varnothing$;

(3) if $s, t \in S, w \in D(s)$, and $t w \notin D(s)$, then $t w=w s$.

Then $S$ is a Coxeter system.

Proof. Conditions (1) and (2) say that $S$ is a system of reflections on $\langle S\rangle$. Put $E(s)=D(s) s$, let $w \in\langle S\rangle$ and let $s \in S$. We first argue by induction on $l_{S}(w)$ that one of the following conditions holds:

(a) $w \in D(s)$,

(b) $w \in E(s)$ and $l_{s}(w s)<l_{s}(w)$.

Namely, write $w=t w_{1}$ for some $t \in S$ and some $w_{1} \in\langle S\rangle$ with $l_{S}\left(w_{1}\right)<$ $l_{s}(w)$. If (a) holds for $w_{1}$ but not for $w$, we get $w=w_{1} s$ by hypothesis, so (b) holds for $w$. If (b) holds for $w_{1}$ we have $l_{s}(w s) \leqslant l_{s}\left(w_{1} s\right)+1<l_{s}(w)$ so that (b) cannot hold for ws. Hence (a) holds for ws so (b) holds for $w$.

As an immediate consequence of (4.3) we have

$$
D(s)=\left\{w \in\langle S\rangle \mid l_{s}(w s)>l_{s}(w)\right\}
$$

for all $s \in S$. Choose $s, t \in S$ and let $\tilde{D}(s, t)$ be the set of all $u \in\langle S\rangle$ for which $l_{S}(u v) \geqslant l_{S}(u)$ for all $v \in\langle s, t\rangle$. Clearly there is at least one element of $\tilde{D}(s, t)$ in each left coset of $\langle s, t\rangle$. Let $u \in \tilde{D}(s, t)$ and $v \in\langle s, t\rangle$; then we argue by induction on $l_{\langle s, t\rangle}(v)$ that

$$
l_{s}(u v)=l_{s}(u)+l_{\langle s, t\rangle}(v) \text {. }
$$

Let $u=s_{1} s_{2} \cdots s_{k}$ and $v=s_{k+1} s_{k+2} \cdots s_{n}$, where $s_{i} \in S(1 \leqslant i \leqslant k)$, 
$s_{i} \in\{s, t\}(k+1<i<n), k=l_{s}(u)$, and $n-k=l_{\langle s, t\rangle}(v)$. We may assume that $n>k$ and, inductively, that $l_{S}\left(u v s_{n}\right)=n-1$. Choose notation so that $s_{n}=s$, and define $w_{n-1}=s_{n-1}, w_{i}=s_{i} w_{i+1}$ for $1 \leqslant i<n-1$. Hence $w_{1}=$ uvs. If $w_{1} \in D(s)$ then $l_{s}\left(w_{1} s\right)>n-1$. Since $u v=w_{1} s$ we get $l_{s}(u v)=n$ as required. Thus we may suppose, by way of contradiction, that $w_{1} \in E(s)$. Clearly, $w_{n-1} \in D(s)$. Hence there exists $j$ such that $w_{j} \in E(s)$ and $w_{j+1} \in$ $D(s)$. Therefore the hypothesis yields $w_{j}=s_{j} w_{j+1}=w_{j+1} s$. If $j>k$ this relation implies that $l_{\langle s, t\rangle}(v)<l_{\langle s, t\rangle}(v s)$, which is not the case, while if $j<k$, it implies that $u \equiv s_{1} s_{2} \cdots s_{j-1} s_{j+1} \cdots s_{k}(\bmod \langle s, t\rangle)$, contrary to our choice of $u \in \tilde{D}(s, t)$. We have therefore established (4.5) which, together with (4.4), shows that $S$ is a pairwise proper system of reflections. Hence $S$ is a Coxeter system.

\section{REFERENCES}

1. N. Bourbaki, Groupes et algèbres de Lie, Chaps. IV, V, VI, Actualités Sci. Indust., No. 1337, Hermann, Paris, 1968.

2. H. S. M. Coxeter, The complete enumeration of finite groups of the form $R_{i}^{2}=\left(R_{i} R_{j}\right)^{k_{v}}=1, \mathrm{~J}$. London Math. Soc. 10 (1935), 21-35.

3. J. Tits, Buildings of spherical type and finite BN pairs, Lecture Notes in Math., vol. 386, Springer-Verlag, New York, 1974.

Department of Mathematics, University of California, Berkeley, California 94720 